\section{Cahiers de Narratologie}

Analyse et théorie narratives

\title{
Les névroses littéraires, laboratoires d'écriture. Camillo Boito, Notte di Natale (1876)
}

\section{Edwige Comoy Fusaro}

\section{OpenEdition}

\section{Journals}

Édition électronique

URL : http://journals.openedition.org/narratologie/5991

DOI : 10.4000/narratologie.5991

ISSN : $1765-307 X$

Éditeur

LIRCES

Référence électronique

Edwige Comoy Fusaro, « Les névroses littéraires, laboratoires d'écriture. Camillo Boito, Notte di Natale (1876) », Cahiers de Narratologie [En ligne], 18 | 2010, mis en ligne le 05 janvier 2011, consulté le 19 avril 2019. URL : http://journals.openedition.org/narratologie/5991; DOI : 10.4000/narratologie.5991

Ce document a été généré automatiquement le 19 avril 2019

\section{(c) (i) (9)}

Cahiers de Narratologie - Analyse et théorie narratives est mis à disposition selon les termes de la licence Creative Commons Attribution - Pas d'Utilisation Commerciale - Pas de Modification 4.0 International. 


\title{
Les névroses littéraires, laboratoires d'écriture. Camillo Boito, Notte di Natale (1876)
}

\author{
Edwige Comoy Fusaro
}

1 Le Risorgimento avait suscité l'émergence d'une littérature empreinte d'une exigence éthique et patriotique, consacrant le triomphe du roman. Avec la proclamation du Royaume d'Italie, cette source d'inspiration privilégiée vient naturellement à manquer. Les écrivains de la génération post-unitaire se trouvent ainsi confrontés au défi de renouveler la littérature. Il s'agit en premier lieu de dépasser le modèle romanesque de Manzoni (Les fiancés), mais aussi d'inventer de nouvelles formes poétiques et narratives. On assiste ainsi à une floraison d'œuvres innovantes et expérimentales, et à un retour du texte court - nouvelles, récits, fragments - notamment au sein du mouvement littéraire et artistique de la Scapigliatura.

2 Tous ces textes ont en commun de faire une place de choix aux maladies nerveuses ${ }^{1}$, qui « contaminent » la littérature de façon presque épidémique, au-delà des mouvements et des genres. Toutefois, les névroses ne sont pas seulement un thème littéraire dominant de la deuxième moitié du $\mathrm{XIX}^{e}$ siècle, elles constituent un véritable bouillon de culture des nouvelles formes de littérature. Parce qu'elles renvoient à tout ce que la médecine de l'époque reléguait hâtivement dans les marges pathologiques du réel connu et que les écrivains eux-mêmes, malgré leur attitude polémique vis-à-vis de la médecine officielle, taxaient de pathologique, elles ouvrent la voie à l'« étrange ». Et c'est parce qu'elles sont mystérieuses (maladies sine materia, d'après la définition originelle de William Cullen) que les névroses ouvrent un nouvel espace littéraire à l'enseigne de l'incertitude, du déséquilibre et de l'inachèvement.

L'hybridation du texte littéraire fait le lit des nouvelles perspectives. A partir de la moitié du siècle, schématiquement, les documents d'« écriture ordinaire » s'invitent au banquet de la littérature, allant parfois même jusqu'à se greffer, tels quels, dans les textes ${ }^{2}$. La présence de ces "greffons » introduit une polyphonie inédite au sein de l'œuvre, ce qui a pour effet immédiat de saper la notion de vérité - tandis que l'effet de réalité s'en trouve 
bien sûr renforcé. La multiplicité des sources supposées authentiques crée le doute dans l'esprit du lecteur, puisque la confrontation des données hétérogènes fait apparaître des contradictions et que, par ailleurs, les prétendus « documents humains » (lettres, journal intime) sont en réalité fictifs : dans la littérature italienne post-unitaire, le "document » inséré dans la trame fictionnelle est un leurre. L'auteur utilise les qualités du texte ordinaire - destiné à une communication privée, dépourvu de visées esthétiques, supposé relater fidèlement la réalité - pour désorienter davantage son lecteur. Ce n'est pas un hasard si tous les textes de cette époque qui proposent un tissu narratif en forme de patchwork présentent un cas de névrose. Au-delà du sujet narratif, c'est une nouvelle dimension "névrotique» du texte qui émerge et bouleverse ses coordonnées: ses instances, ses modalités d'expression, ses enjeux, sa fonction, sa portée.

Le corpus de Camillo Boito ${ }^{3}$ constitue un bon point d'ancrage pour observer ces nouveaux textes informés par les névroses. Le discours du protagoniste y est régulièrement démenti par les autres instances ou par la structure même du texte, au moyen d'un morceau narratif exogène, du discours des autres personnages ou, plus subtilement, du montage savamment incohérent du récit. La nouvelle Notte di Natale (Nuit de Noël) ${ }^{4}$ nous en fournit un exemple.

Giorgio, le protagoniste, vivait heureux à Turin avec sa sœur jumelle Emilia, sa nièce Giorgetta et leur vieille nourrice Maria, jusqu'à ce que l'enfant ne meure des suites d'une diphtérie, suivie de près dans la tombe par sa mère, Emilia. Bouleversé, Giorgio contracte une étrange " maladie de l'estomac ${ }^{5}$ » qui lui ôte l'appétit. Accablé de douleur, il quitte sa ville natale pour parcourir l'Italie. A Milan, il rencontre une modiste dont le sourire lui rappelle celui de sa chère disparue. La nuit de Noël, il l'invite dans sa chambre d'hôtel et, pour la première fois depuis la mort d'Emilia, parvient à manger normalement - et même à faire bombance. La modiste s'endort et Giorgio, fasciné par ses dents magnifiques, lui en arrache une dans son sommeil, puis retourne aussitôt à Turin. Pendant les trois premiers jours qui suivent son retour dans la maison natale, Giorgio écrit ses " vilaines confessions ${ }^{6}$ " qu'il remet ensuite à Maria. Celle-ci remarque qu'il se porte mieux mais le jour suivant, une forte fièvre le cloue au lit: il ne peut plus rien ingérer et commence à délirer. Le septième jour, la modiste se présente chez lui pour réclamer de l'argent en échange de sa dent: en la voyant sourire (un trou noir à la place d'une dent), Giorgio pousse un cri d'effroi et rend son dernier soupir.

6 La nouvelle comporte deux types de récit, en apparence deux écrits ordinaires. Le tableau central - les pages manuscrites de Giorgio - fournit la vision « intérieure », « historique » du personnage névrosé, tandis qu'en ouverture et en clôture de nouvelle se trouve un texte-cadre, périphérique, de la main de Maria, personnage indiscutablement sain d'esprit, porte-parole d'un regard " extérieur », « clinique », prétendument "normal ». Dans son manuscrit, Giorgio évoque sa relation avec la modiste. Le personnage-narrateur oscille manifestement entre des moments de lucidité et des moments d'aveuglement névrotique. Quand il est lucide, force lui est de constater que la modiste est laide

Elle n'était pas si mal de sa personne, mais son visage avait des traits vulgaires, sa peau était rugueuse, mouchetée de petites taches jaunes, elle avait des cernes verdâtres et son front était creusé de fines rides parallèles ${ }^{7}$;

Elle est donc très différente de sa sœur, qui avait au contraire des cernes bleuâtres et un teint soyeux ${ }^{8}$. En revanche, dans les moments d'aveuglement, la ressemblance de la modiste milanaise avec la regrettée Emilia semble incontestable : 
[...] ses lèvres couleur corail ouvertes en arc encadraient la blancheur de neige de ses dents parfaites. Elle ressemblait, en effet, à Emilia9 . «trivial » et, qui plus est, cupide et sans vergogne. Les deux brefs morceaux de texte qui sont de sa plume ont donc pour fonction de mettre en garde contre le discours douteux des confessions de Giorgio, qui portent la marque du déséquilibre mental. Le texte central opère néanmoins une percée dans un univers subjectif auquel seule la pathologie nerveuse donne accès. Boito offre une tribune à la parole du malade: une parole qui propose une autre vision des choses, déformée, certes, mais qui se trouve receler des ressources cognitives insoupçonnées.

En premier lieu, la vision du malade s'exerce également «en dedans » et « en arrière » (puisqu'elle tient compte de son idiosyncrasie et appréhende le sujet dans sa diachronie), tandis que l'œil « médical », relayé par le texte-cadre de la nourrice, se borne à la surface $\mathrm{du}$ corps et à son état présent ${ }^{10}$. Les " confessions » de Giorgio font donc la lumière sur des éléments qui échappent à l'observation extérieure, "clinique ", et ne peuvent s'exprimer que dans un « récit »: elles nous parlent de l'affection de Giorgio pour sa sœur et de la douleur inconsolable de sa perte; en revanche, le regard objectif ne peut saisir que ses symptômes sensibles. L'insuffisance du discours de Maria et la richesse du récit «névrotique » sont par ailleurs suggérées par la stratégie mise en œuvre par l'auteur pour dérouter le lecteur au cœur de la narration, notamment dans les profondeurs analogiques des mots, des images et des symptômes. Ainsi la thèse de la dissemblance des deux femmes (Emilia / modiste) n'est-elle pas plus crédible que celle de leur ressemblance, tout comme la thèse de la maladie présente de Giorgio n'est pas plus fiable que celle de sa bonne santé passée - c'est ce que nous allons démontrer. La prétention à l'omniscience du modèle de pensée anatomique et rationnel est incriminée au profit d'une approche alternative poétique, préfreudienne et consciente de ses limites. La névrose qui informe le texte de Boito se présente ainsi comme le meilleur outil de sonde d'une fabula vouée à conserver une part irréductible de mystère et, dans le même temps, comme l'expression la plus adaptée d'une nouvelle approche du réel et de la littérature : partielle, énigmatique, irrationnelle.

10 Les douleurs gastriques de Giorgio ont débuté après la mort d'Emilia, de sorte que le lecteur est naturellement porté à croire que c'est la perte de l'être cher qui l'a fait tomber malade. Cependant cet événement tragique n'est que l'élément déclencheur d'un état névrotique préexistant. En effet, l'idylle familiale que Giorgio évoque en se remémorant les belles soirées passées auprès de sa sœur et de sa fille Giorgetta dessine, en creux, un univers affectif morbide. Le milieu familial du personnage est anormal : il n'est pas fait mention des parents de Giorgio et Emilia, remplacés par l'ersatz de mère qu'est la nourrice ; il n'est pas fait mention non plus du père de Giorgetta. Le trio familial GiorgioEmilia-Giorgetta entretient par ailleurs un rapport nettement fusionnel: Maria nivelle leurs différences d'âge et de sexe en parlant d'eux comme de «trois chers enfants ${ }^{11}$ »; en outre, Giorgio et Emilia sont jumeaux et Giorgetta se présente à la fois comme une Emilia miniature, de par sa ressemblance physique avec sa mère ${ }^{12}$, et comme un Giorgio miniature, en raison de la ressemblance de leurs prénoms. De plus, Giorgio est le seul homme du foyer mais c'est un homme apparemment asexué et son rôle officiel de frère et d'oncle cède de toute évidence la place au statut affectif de fils, vis-à-vis de Maria, bien sûr, mais aussi vis-à-vis d'Emilia : «Tu as besoin qu'on prenne grand soin de toi », lui dit Emilia avant de rendre son dernier soupirit ${ }^{13}$. 
11 Dans l'esprit malade de Giorgio, la mort d'Emilia équivaut donc à la perte de la mère ; elle équivaut également à la perte du meilleur de lui-même car, conformément à la symbolique de la gémellité comme système bipolaire, Emilia et Giorgio sont en réalité deux natures opposées : Emilia est adulte et bonne (elle est mère) tandis que Giorgio est, symboliquement, un enfant méchant. Les pathologies qui les affligent ont une valeur métaphorique incontestable : Emilia est emportée par la diphtérie, une maladie des voies supérieures (aériennes), tandis que Giorgio contracte une maladie des voies basses (digestives). En toute logique, la jeune femme se spiritualise dans l'agonie tandis que la maladie plonge Giorgio dans la turpitude. La mort d'Emilia est donc une amputation pour le protagoniste, qui s'en trouve totalement décentré, déséquilibré : à ceci près que son absence «préalable » d'autonomie et d'auto-centralité est déjà, en soi, indiscutablement névrotique ${ }^{14}$.

Le discours superficiel selon lequel la mort d'Emilia déclencherait le mal de Giorgio est donc bien erroné. Mais ce discours erroné, porteur du regard «clinique» de Maria, et relayé par les passages de lucidité apparente du manuscrit de Giorgio (ceux où la dissemblance de la défunte sœur et de la modiste est frappante), recouvre un ensemble bien plus large. Ainsi la vie de Giorgio apparaît-elle coupée en deux parties antithétiques, de sorte que la vie sans Emilia se présente comme une parodie infernale de la vie avec Emilia. Pour s'en convaincre, il suffit de confronter les éléments descriptifs du couple antinomique Emilia / la modiste, qui incarnent le monde de Giorgio avant et après la perte : la voix d'Emilia est douce et limpide, celle de la modiste est rauque ${ }^{15}$; les mains d'Emilia sont roses et délicates, celles de la modiste sont rugueuses ${ }^{16}$; Emilia a le teint diaphane et des cernes délicatement bleutées, la modiste a la peau mouchetée de taches jaunes et des cernes verdâtres ${ }^{17}$; par ailleurs, Emilia porte des valeurs morales et spirituelles, tandis que la modiste est une femme vulgaire aux valeurs purement matérielles ${ }^{18}$. Enfin, les passages consacrés à Emilia sont dominés par l'image récurrente de la perle ${ }^{19}$, pierre lunaire et féminine en raison de la douceur de son éclat, dont la forme sphérique évoque la perfection. La blancheur (du teint, de l'ivoire des dents, de la perle) connote efficacement le portrait angélique d'Emilia. En revanche, les images associées à la modiste composent un décor infernal. L'obscurité en est un premier élément : Giorgio ne rencontre la modiste que le soir ou la nuit, quand il fait sombre ${ }^{20}$. En conséquence, toutes les lumières présentes lors de leurs rencontres sont artificielles. Boito insiste sur le flamboiement des flammes, auquel fait écho le miroitement des bijoux ${ }^{21}$, notamment dans la scène proto-décadente dans la chambre d'hôtel :

La chambre de l'hôtel Cavour, où j'habitais depuis plusieurs jours, était bien chauffée : les flammes dansaient dans la cheminée. Je fis allumer deux candélabres et le lustre qui pendait de la voûte. Dans cette grande lumière les murs d'un jaune d'or à fleurs rouges juraient ${ }^{22}$.

Il y avait de plus en plus de verres sur la table, tous de formes différentes. Chacune des chandelles projetait sur leurs facettes une lame brillante comme de petites étincelles électriques. [...] Le vin ressemblait à des pierres précieuses liquéfiées, améthystes, rubis, topazes ${ }^{23}$.

Le repas de Noël se transforme en ripaille : il subit un renversement parodique proche de la profanation. Giorgio et la modiste dégustent en effet le faisan aux truffes «dans un recueillement religieux $^{24} »$ : les plaisirs de la chair et du ventre remplacent le recueillement de l'Avent, la joie de la fête religieuse. La disparition d'Emilia et Giorgetta précipite donc Giorgio dans un monde à l'envers, pathologique, pernicieux, comme s'il n'était pas capable, par lui-même, de vivre dans la normalité et en accord avec la morale. 
Le milieu infernal qui caractérise la vie après Emilia est proprement spéculaire de l'autoportrait suggéré de Giorgio en enfant mauvais.

Cependant, la dichotomie manichéenne qui sépare, au niveau fictionnel, la vie idyllique et la vie dyonisiaque de Giorgio et, au niveau narratif, le regard médical de Giorgio-lucide et de Maria et la vision déformée de Giorgio-névrosé, - cette forte dichotomie est fallacieuse, comme l'indiquent l'anormalité de la vie de Giorgio avant la mort d'Emilia et, ce que nous allons observer à présent, la question problématique de la ressemblance entre Emilia et la modiste.

fenture. Cet élément «fondamental » est polysémique $^{25}$ : il renvoie à la pureté (grâce aux attributs de blancheur, de régularité, de transparence ${ }^{26}$ ) mais aussi à la dévoration, à la rapacité, à la déglutition, donc aux fonctions digestives du corps et, par extension, à la sexualité et à la mort - « le buccal étant l'emblème régressé du sexuel $\aleph^{27}$. Or, Giorgio est aveugle au deuxième sens de l'image ${ }^{28}$. Chez la modiste, les dents sont interprétées par Giorgio comme un signe entièrement positif alors que l'insistance sur son avidité et sa vulgarité ( j'ai le ventre plein ", s'exclame-t-elle sans grâce avant de réclamer du champagne ${ }^{29}$ ) induit tout naturellement à privilégier, au contraire, la deuxième valeur de l'image, c'est-à-dire sa connotation abyssale. Vice-versa, Giorgio occulte totalement cette même connotation chez Emilia, qu'il idéalise sans voir en elle sa matérialité charnelle, soumise à la putrescibilité et elle-même porteuse d'une tension dévorante - un corps qui dévore et qui sera dévoré à son tour ${ }^{30}$. Il est ainsi très troublant qu'Emilia ait les yeux noirs, exactement comme la modiste :

Ses yeux noirs étaient ouverts; ses cheveux noirs entouraient son blanc visage ; au milieu de cette blancheur lugubre et de cette noirceur funèbre se détachait le rose de ses lèvres entrouvertes, laissant voir les dents plus blanches encore que le front de cette pauvre morte ${ }^{31}$.

Il appert que Giorgio, dès avant la perte d'Emilia, était bel et bien atteint d'une " cécité » métaphorique, partielle, d'un " aveuglement » névrotique, un refus de voir la réalité dans sa totalitéz ${ }^{32}$. La mort de la sœur chérie ne fait donc que rendre visible un processus délétère antérieur et sans issue : d'ailleurs, le retour au foyer natal après la malheureuse expérience milanaise (retour symbolique au giron maternel ${ }^{33}$ ) ne tient pas ses promesses de bonheur; le bonheur est perdu d'avance et sa perte ne remonte pas à la mort d'Emilia mais, très probablement, à la séparation d'avec la mère nourricière, que la bonne Maria ne peut suffire à suppléer pleinement. La nostalgie du sein maternel est en effet suggérée par le symptôme névrotique qui exauce symboliquement son désir de régression: souffrant d'inappétence, Giorgio ne se nourrit que de substances non solides, puis n'accepte plus que le lait avant de sombrer, plus radicalement, dans l'anorexie totale ${ }^{34}$. La tolérance exclusive des nourritures molles et liquides le ramène au temps de la première phase du stade buccal (la succion), temps du bonheur complet où l'absence de dents ne l'avait pas encore condamné au croquage, c'est-à-dire à la dévoration.

La période prétendument normale, heureuse et saine de la vie de Giorgio s'avère donc avoir été indéniablement pathologique. Inversement, la période de sa vie inaugurée par la disparition d'Emilia et Giorgetta place le personnage dans une position inédite de normalité, de « santé » : libre qu'il est désormais de s'ouvrir à l'autre sexe pour fonder luimême une famille. Cependant, Giorgio est manifestement incapable de vivre dans cet état de "normalité » puisque sa fréquentation de la modiste se solde par une aggravation de son symptôme. En somme, le jeune homme n'a jamais été aussi normal que lorsqu'il a 
développé une maladie psychosomatique ; globalement, il a toujours été malade mais c'est paradoxalement dans la condition d'anormalité (en faisant ménage avec sa sœur et en renonçant à toute activité sexuelle ${ }^{35}$ ) qu'il trouve le bonheur.

On constate toutefois que ce résultat est le fruit d'une exégèse très serrée : le texte, en luimême, se limite à relater des points de vue fragmentaires et captieux. En effet, les confessions de Giorgio produisent une version altérée des faits, déformée par le prisme de la névrose ; quant au texte-cadre, supposé objectif, il n'est guère plus fiable que le récit du personnage névrosé puisque Maria partage l'avis de Giorgio quant à la normalité de sa vie avant la mort d'Emilia. Il n'est donc plus question de " vérité » : plus exactement, le texte littéraire offre un espace d'expression à « une » vérité cryptée, celle du malade.

La nouvelle, dans son ensemble, se présente ainsi comme une sorte d'anamorphose, à l'image des symptômes névrotiques. De ce fait, une nouvelle fonction est accordée aux narrateurs, porteurs d'une vision subjective du réel, et au lecteur, qui est invité à douter des vérités apparentes et à décoder les énigmes du texte. Le texte, quant à lui, se présente alors comme le morceau incomplet d'un récit truffé de leurres. Ces nouvelles modalités impliquent en effet l'émergence d'un hors-texte virtuel. En amont se trouvent les nondits du texte ${ }^{36}$, dont l'analyse soupçonne l'importance décisive sans toutefois qu'aucune garantie d'exactitude ne soit jamais concédée, et en aval se trouve la résolution de l'intrigue, que la fin ouverte rend à jamais inaccessible ${ }^{37}$. L'attente du lecteur est donc irrémédiablement frustrée. C'est bien là un but essentiel, semble-t-il, de ces œuvres. Loin de chercher l'assentiment du lecteur, comme c'était le cas de Manzoni et, parmi les contemporains de Boito, des adeptes du naturalisme ${ }^{38}$, notre auteur s'efforce au contraire de le désorienter, de mettre en cause toutes ses certitudes. On ne demande plus au lecteur d'être réceptif (donc passif), on exige de lui qu'il soit autonome et actif, on lui impose d'enquêter sur la piste des indices semés par l'auteur.

Le climat d'incertitude créé par les névroses littéraires achemine le texte aux confins du genre fantastique ${ }^{39}$. L'œuvre, comme le mal, se dérobe, on ne peut pas l'appréhender dans sa totalité, on peut seulement en saisir des bribes, des traces fugaces, des signes plus ou moins trompeurs. L'auteur renonce à l'omniscience et à la prise de parti ; le lecteur demeure dans l'hésitation et ses talents d'analyste ne suffisent pas à compléter les lacunes du récit ni à déchiffrer ses réseaux analogiques et métaphoriques - son enquête versant inévitablement dans la spéculation; le texte est énigmatique, ouvert, fragmentaire et centrifuge, irrémédiablement résistant à l'analyse rationnelle.

21 Ces nouvelles formes de mise en récit auraient-elles pu voir le jour sans les névroses? Certes non. Les insaisissables névroses constituent le creuset de ces évolutions : elles seules donnent accès à une réalité complexe, polycentrée, absconse, du moins au niveau de la conscience éveillée (dans d'autres états de conscience, le rêve y pourvoit efficacement). Elles ouvrent une brèche dans la conception "positive» du monde et de l'homme (et de la littérature), brèche qui fait entrevoir une dimension qui tient en échec l'outillage mental et instrumental de la médecine clinique. L'incertitude azimutale de cette nouvelle littérature conduira à la cécité partielle (partiale) des personnages de Tozzi et sa poétique des misteriosi atti nostri, à la crise identitaire des personnages de Svevo et Pirandello, à l'univers macaronique de Gadda; dans le domaine médical, elle annonce bien sûr la psychanalyse puisque les névroses exigent une mise en récit, c'est-à-dire une approche littéraire. En cette période riche des ferments de la modernité du XXe siècle et de la révolution freudienne, mais qui reste solidement attachée au mythe de l'omnipotence cognitive de la science et de l'omnipotence expressive de la littérature, les 
névroses sont comme des vecteurs de thérapie génique. Ce sont elles qui permettent le transfert du " gène » de la modernité dans la cellule-texte (et, à plus grande échelle, dans l'organisme-littérature) : par leur charge pathologique, elles portent encore la marque de leur temps - la deuxième moitié $\mathrm{du} \mathrm{XIX}^{\mathrm{e}}$ siècle-, dominé par une doxa exceptionnellement normative; mais sous la marque infamante, nous avons bien déjà affaire à des formes embryonnaires de fragmentisme, de monologue intérieur, de textes métalittéraires aussi - de formes, en somme, dont on ne mesurera pleinement la pertinence qu'au $\mathrm{XX}^{\mathrm{e}}$ siècle, une fois l' "épidémie » enfin terrassée. En attendant, la génération post-unitaire relève admirablement le défi d'inventer une littérature alternative à l'imposant modèle manzonien.

\section{NOTES}

1. La perspective critique retenue ici consiste à retenir dans le domaine des névroses ce qui était considéré comme tel à l'époque: un domaine bien plus vaste et bien plus flou qu'il ne l'est aujourd'hui.

2. Giuseppe Zaccaria voit dans l'ouverture des frontières du littéraire l'une des caractéristiques majeures de la littérature de la deuxième moitié du XIXe siècle (Giuseppe Zaccaria, La fabbrica del romanzo (1861-1914), Genève-Paris, Slatkine, 1984). Cette thèse est reprise par Tommaso Pomilio (Tommaso Pomilio, Paradigmi atopici: Milano 1860-1881, in Letteratura italiana e utopia, Annali del Dipartimento di Italianistica dell'Università La Sapienza di Roma, Roma, Editori Riuniti, 1995). Sur la notion d'« écriture ordinaire » comme pratique d'écriture n'appartenant pas à la «fonction auteur ", cf. la contribution de Philippe Artières, qui emprunte lui-même l'expression aux travaux de Daniel Fabre (Philippe Artières, «Ecritures ordinaires du sida", in Littérature et médecine ou les pouvoirs du récit, actes du colloque des 24-25 mars 2000, sous la direction scientifique de G. Danou, Paris, Bpi/Centre Pompidou, 2001).

3. Camillo Boito (Rome 1836-Milan 1914) fait ses études à l'Académie des Beaux Arts de Venise et suit à Padoue une spécialisation qui lui permet de se lancer dans l'architecture. Il mène alors de front son enseignement à l'Académie de Venise, où il engagé dès l'âge de vingt ans, sa carrière d'architecte et sa collaboration régulière aux revues périodiques. En 1860 il obtient un poste de professeur d'architecture à l'Académie milanaise de Brera, où il rejoint son frère cadet Arrigo (Enrico à l'état civil). Au début des années 1870, il se lance dans la littérature. Il publie d'abord ses nouvelles en revue, puis les réunit dans deux recueils : Storielle vane (1876) et Senso. Nuove storielle vane (1883). C'est néanmoins son activité d'architecte qui lui vaut sa renommée, tandis que, de son vivant et pendant une longue période de l'historiographie littéraire, ses œuvres littéraires sont totalement occultées par celles de son frère Arrigo, figure de proue de la Scapigliatura. On doit la dernière édition de ses œuvres à Chiara Cretella (Camillo Boito, Storielle vane, Bologna, Pendragon, 2007 ; Id., Senso. Nuove storielle vane, Ravenna, Allori, 2005).

4. La nouvelle parut d'abord dans la revue florentine « Nuova Antologia di scienze, lettere ed arti » en janvier 1876, accompagnée du sous-titre Storiella vana; puis elle fut insérée, sans soustitre, dans les Storielle vane auprès de l'éditeur milanais Treves. Notre texte de référence est compris dans Camillo Boito, Il collare di Budda, Firenze, Passigli, 1994, pp. 63-84. En français, cf. Id., Histoires vaines, trad. Karin Dubois, Toulouse, Ombres, 1999, pp. 7-23. Les passages du texte cités en français dans cet article sont toutefois traduits par nos soins. 
5. « malattia di stomaco " (Camillo Boito, Notte di Natale, cit., p. 82).

6. «le mie brutte confessioni » (Ivi, p. 83).

7. «La personcina non c'era male, ma il volto aveva de' lineamenti triviali, la pelle ruvida chiazzata di macchiette gialle, le occhiaie verdastre, la fronte solcata di sottili rughe parallele » (Ivi, p. 76).

8. «i lividori sotto gli occhi [...] di un azzurretto fino », « il morbido incarnato » (Ivi, p. 80).

9. «le labbra coralline ed aperte in arco facevano cornice al candore niveo dei denti perfetti. Somigliava, in fatti, all'Emilia » (Ivi, p. 77).

10. De fait, la médecine s'avère impuissante face aux maux dont il souffre. Dans son manuscrit, Giorgio affirme avoir ingéré des doses excessives de pepsine, sans aucun effet bénéfique sur ses douleurs d'estomac (« La pepsina, presa in quegli ultimi giorni senza misura e senza ordine, [...] non era riescita ad altro sino allora che a martoriare peggio che mai il mio stomaco », Ivi, p. 66). Il affirme par ailleurs avoir consulté d'illustres médecins en vain («Avevo già da molti mesi esaurito tutti i medicamenti possibili, persino, con mia onta, quelli delle quarte pagine dei giornali ; avevo interrogato i medici illustri di Berlino e di Parigi », Ivi, p. 74). L'impuissance de la médecine est l'un des signes les plus irréfutables de la présence d'une forme de névrose.

11. " quei tre cari fanciulli » (Ivi, p. 63).

12. «tenevo nel portafogli quattro diversi ritratti dell'Emilia, e tre anche della Giorgetta, i quali parevano tre immagini dell'Emilia fanciulla » (Ivi, p. 68).

13. « Hai bisogno di molte cure » (Ivi, p. 81).

14. Sur le topos du personnage centrifuge, cf. Vittorio Roda, Homo duplex. Scomposizioni dell'io nella letteratura italiana moderna, Bologna, Il Mulino, 1991; Id., I fantasmi della ragione. Fantastico, scienza e fantascienza nella letteratura italiana fra Otto e Novecento, Napoli, Liguori, 1996.

15. «la sua voce dolce ", « la voce limpida» (Camillo Boito, Notte di Natale, cit., pp. 69, 79) vs «con voce rauca » (Ivi, p. 76).

16. « le sue mani rosee e delicate» (Ivi, p. 70) vs « le mani aspre» (Ivi, p. 76).

17. «i lividori sotto gli occhi [...] di un azzurretto fino », « il morbido incarnato », « una pallida tinta diafana di avorio » (Ivi, p. 80) vs « la pelle ruvida chiazzata di macchiette gialle, le occhiaie verdastre » (Ivi, p. 76).

18. «La soavità di quella indole dolce [Emilia], pronta al bene, dimentica sempre di sé, ingenua, gentile, saggia, si purificava in natura d'angelo. Di mano in mano che il male le rodeva i visceri il suo spirito si alzava a Dio » vs la cupidité et la trivialité de la modiste.

19. Emilia avait offert à Giorgio une épingle à cravatte ornée d'une perle pour sa fête et le dernier cadeau de Giorgio à sa sœur était un collier de perles (cf. Ivi, pp. 70 et 84).

20. Les premières fois qu'il la voit, c'est «nell'ora già buia in cui si accendono i fanali » (Ivi, p. 68); le jour où il décide de la suivre, il la voit entrer par une porte et « perdersi tosto nel buio fitto di un andito » (Ivi, p. 69) ; le soir de Noël, il aperçoit son ombre « in fondo al nero andito » (Ivi, p. 70) et, lorsqu'il s'approche pour lui parler, elle recule, « dileguandosi nelle tenebre » (Ivi, p. 71).

21. Le soir où Giorgio va l'aborder, la modiste est en train d'admirer la vitrine d'un orfèvre : «Stavano accendendo i lumi, e gli ori scintillavano, e i diamanti brillavano » (Ivi, p. 69).

22. «La stanza dell'albergo Cavour, dove abitavo da parecchi giorni, era ben calda: le fiamme guizzavano nel caminetto. Feci accendere due candelabri e il lampadario che pendeva dalla vàlta. In quella gran luce le pareti d'un giallo d'oro a fiori rossi stridevano » (Ivi, p. 73).

23. «La varietà dei bicchieri era andata crescendo. Ogni candela mandava sulle loro faccette una striscia lucida come di piccole scintille elettriche. [...] Il vino somigliava a pietre preziose liquefatte, amatiste, rubini, topazi » (Ivi, p. 74).

24. "mangiavamo in religioso raccoglimento » (Ivi, p. 74).

25. Selon Bachelard, l'image " archaïque » ou fondamentale se couvre facilement de "valeurs contraires». Cf., par exemple, ses considérations sur «la noirceur secrète du lait» dans un 
sonnet d'Audiberti (Gaston Bachelard, La terre et les rêveries du repos. Essai sur les images de l'intimité , Paris, José Corti, 1948, pp. 59, 23 et suivantes).

26. Cf. la description des dents de la modiste : «Erano tutti uguali, tutti piantati regolarmente ; quelli di sopra un poco più grandi e tanto sottili, che parevano trasparenti » (Camillo Boito, Notte di Natale, cit., p. 78).

27. Gilbert Durand, Les structures anthropologiques de l'imaginaire. Introduction à l'archétypologie générale, Paris, Dunod, 1984, p. 129. "Le ventre sous son double aspect, digestif et sexuel, est donc un microcosme du gouffre, est symbole d'une chute en miniature », le tube digestif " est l'abîme euphémique et concrétisé » (Ivi, pp. 131 et 133). « Essentiel à l'être, l'aliment représente à la fois un besoin et un danger - lequel est marqué dans les mythes de notre civilisation, dès le départ, par l'acte de dévorer le fruit de l'arbre du bien et du mal, modèle de toutes les transgressions d'interdit » (Marie-Claire Bancquart, Fin de siècle gourmande (1880-1900), Paris, PUF, 2001, p. XI).

28. Sa cécité partielle est d'ailleurs subtilement annoncée dans le tableau liminaire qui plante le décor, où le brouillard épais provoque un engourdissement sensoriel de l'ouïe et de la vue : «Si navigava nella nube, bagnati, intirizziti, sospettando di essere diventati ciechi e sordi » (Camillo Boito, Notte di Natale, cit., p. 64).

29. « ho la pancia piena » (Ivi, p. 75).

30. «Manger, c'est nier, c'est dévorer, c'est être cruel, c'est être assassin. Donc exister, c'est être cruel et assassin... L'acide mange, et l'alcali aussi ; la plante mange, l'animal mange, l'homme mange, tout mange » (Pierre Leroux, La Grève de Samarez, livre II, in Gaston Bachelard, La terre et les rêveries du repos, cit., pp. 63-64).

31. «Gli occhi neri erano aperti ; i capelli neri circondavano la faccia bianca ; le labbra socchiuse spiccavano rosee in mezzo a quel bianco lugubre e a quel nero funereo, lasciando vedere i denti ancora più candidi della fronte di quella povera morta » (Camillo Boito, Notte di Natale, cit., p. 81).

32. C'est significativement la révélation irréfutable de la dissemblance (grâce à la dent manquante qui interdit désormais tout rapprochement entre la modiste et Emilia), c'est-à-dire la pleine vision du monde à l'envers, définitivement amputé de son principe spirituel, qui porte le coup fatal à Giorgio.

33. "Le retour au pays natal, la rentrée dans la maison natale, avec tout l'onirisme qui le dynamise, a été caractérisé par la psychanalyse classique comme un retour à la mère. [...] L'intimité de la maison bien fermée, bien protégée appelle tout naturellement les intimités plus grandes, en particulier l'intimité d'abord du giron maternel, ensuite du sein maternel » (Gaston Bachelard, La terre et les rêveries du repos, cit., pp. 121-122).

34. Au début de son manuscrit (entre la mort d'Emilia et la rencontre de la modiste), Giorgio écrit qu'il devait se nourrir «di brodi allungati, di latte, di caffè, di pezzettini di carne sanguinolenta ». Le quatrième jour après son retour à Turin, « [i]l signor Giorgio non poté più inghiottire nulla, neppure il latte allungato » (Camillo Boito, Notte di Natale, cit., pp. 74, 83).

35. D'après la littérature médicale de l'époque, la continence était un trait pathologique chez les hommes (à moins qu'ils ne fussent voués au sacerdoce) : "Sans aucun doute, l'homme a un besoin sexuel plus vif que la femme. [...] En tout cas, l'homme qui fuit la femme et la femme qui court après la jouissance sexuelle sont des phénomènes anormaux » (Richard von Krafft-Ebing, Psychopatia sexualis [1888], trad. fr. René Lobstein, édition refondue par Albert Moll, Paris, Editions Climats et Librairie Thierry Garnier, 1990, p. 24).

36. Plusieurs autres auteurs placent la racine du mal en amont de l'histoire narrée, en deçà du récit, comme Luigi Gualdo dans Une ressemblance (1874) ou Igino Ugo Tarchetti dans Storia di una gamba (1869). Il s'agit de suggérer l'impossibilité de saisir la vérité de la maladie. Ainsi, dans Une ressemblance, l'origine affichée du mal (la rupture d'avec Annetta) est de toute évidence 
illusoire, cependant seule l'analyse fait le jour sur l'origine supposée réelle du mal (la séparation d'avec la mère).

37. Dans Notte di Natale, Maria nous apprend que Giorgio succombe à ses maux mais dans d'autres textes, c'est l'incertitude qui prédomine. Cf. notamment Macchia grigia (1877), publié significativement un an après Notte di Natale: la nouvelle se présente comme une longue lettre que le narrateur écrit à l'attention des médecins qu'il va consulter pour qu'ils le guérissent d'un scotome (une tache qui entrave son champ visuel). Le manuscrit retrace son histoire de sa maladie - nous avons donc affaire à une anamnèse (subjective) spontanée, doublée d'une description très littéraire du scotome. Ici, cependant, pas de nourrice, pas de narrateur extérieur qui nous fournisse un autre point de vue; pas de fin non plus, puisque la lettre s'achève la veille $\mathrm{du}$ jour où le personnage va la remettre aux médecins. Le devenir du personnage repose donc entre les mains des médecins, dont il est peu crédible qu'ils parviendront à le soigner d'un mal qui n'est qu'un symptôme névrotique et qui prend sa source dans la mauvaise conscience. En effet, quelques lignes avant de conclure son manuscrit, le narrateur découvre enfin la vraie nature de la forme hideuse qui fait obstacle à sa vision: "È il mio vecchio, il mio terribile vecchio! » (Camillo Boito, Macchia grigia, in Id., Il collare di Budda, cit., p. 61). Il s'agit du vieux père désespéré de la jeune fille qu'il avait séduite et abandonnée, qui s'était donné la mort. Que peuvent les médecins de l'époque sur le sentiment de culpabilité ?

38. Dans Giacinta (1879), par exemple, dont Luigi Capuana voulait qu'il fût le premier roman réaliste italien, la maladie de l'héroïne est expliquée point par point : rien ne résiste aux facultés analytiques et expressives de l'« écrivain-anatomiste ", et aucun mystère ne subsiste pour le lecteur.

39. Le frère de Camillo Boito, Arrigo, en fournit un exemple avec Il pugno chiuso (1870). La nouvelle s'achève sans que la lumière soit faite sur la nature exacte des faits narrés. Le texte refuse de prendre parti entre une interprétation rationnelle (et médicale : la pathologie mentale) et une interprétation surnaturelle, toutes deux équitablement représentées. Cf. Arrigo Boito, Poesie e racconti, a cura di Rodolfo Quadrelli, Milano, Mondadori, 1981. En français, cf. Id., Idées fixes, trop fixes, trad. fr. Olivier Favier, Paris, Les Editions du Sonneur, 2007.

\section{RÉSUMÉS}

Camillo Boito est traditionnellement jugé, à tort, comme un auteur mineur de la mouvance littéraire et artistique de la Scapigliatura. Dans Notte di Natale, il invente au contraire des modalités narratives inédites et particulièrement suggestives. L'échec inattendu de l'œil clinique au profit de la vision névrotique participe de la révolution épistémologique qui fait le lit de la modernité littéraire du $\mathrm{XX}^{\mathrm{e}}$ siècle et de l'essor de la psychanalyse

È del tutto ingiusta la tradizionale collocazione di Camillo Boito fra gli autori minori della Scapigliatura. In Notte di Natale, egli inventa delle modalità narrative inedite e particolarmente suggestive. L'inaspettato fallimento dell'occhio clinico a favore della visione nevrotica partecipa della rivoluzione epistemologica che precorre la modernità letteraria del Novecento e l'avvio della psicoanalisi. 
INDEX

Index chronologique : XIXe siècle

Mots-clés : littérature italienne, Scapigliatura, névrose, Camillo Boito

Index géographique : Italie

\section{AUTEUR}

\section{EDWIGE COMOY FUSARO}

Maître de conférences en Langue, littérature et civilisation italiennes à l'Université Nice Sophia Antipolis. Elle est rattachée au CIRCPLES et fait partie du comité de rédaction des Cahiers de narratologie. Elle a publié La nevrosi tra medicina e letteratura. Approccio epistemologico alle malattie nervose nella narrativa italiana (1865-1922), préfacé par F. Livi, Firenze, Polistampa, « Biblioteca di Medicina e Storia », 2007, 463 p., et Forme e figure dell'alterità. Studi su De Amicis, Capuana e Camillo Boito, Ravenna, Giorgio Pozzi editore, « Gallica-Italica », 2009, 238 p. 